\title{
Advances in Clayff Molecular Simulation of Layered and Nanoporous Materials and their Aqueous Interfaces
}

\author{
Randall T. Cygan, ${ }^{\dagger}$ Jeffery A. Greathouse, ${ }^{\dagger}$ and Andrey G. Kalinichev ${ }^{\ddagger}$ \\ ${ }^{\dagger}$ Geochemistry Department, Sandia National Laboratories, Albuquerque, New Mexico, 87185- \\ 0754, United States \\ Laboratoire SUBATECH (UMR 6457 - Institut Mines-Télécom Atlantique, Université de \\ Nantes, CNRS/IN2P3), 44307, Nantes, France
}

\section{Supporting Information}

Parameters for the Clayff interatomic potentials ${ }^{1}$ are provided in Tables S1 and S2. Any differences in parameter values from the original publication are due to reporting of significant digits, roundoff errors, or corrections in transposition of digits. Energy values are presented in $\mathrm{kcal} / \mathrm{mol}$, distances in Ångstroms, and angles in degrees. Note that values for bond-angle bend force constants incorporate radian units, and conversion between degrees and radians is accommodated in the force constant value. Table S1 includes nonbonded interaction parameters using $\varepsilon$ and $\sigma$ convention for the Lennard-Jones (L-J) potential. The original Clayff publication reported values for L-J potentials using the alternative $D_{o}$ and $R_{o}$ convention where $D_{o}=\varepsilon$ and $R_{\mathrm{o}}$ $=2^{1 / 6} \sigma$.

Total potential energy of a molecular system is represented in Clayff as the summation of contributing energy components as described by Equation S1:

$$
E_{\text {Total }}=E_{\text {Coul }}+E_{V D W}+E_{\text {Bond }}+\cdots
$$

The total energy includes contributions from Coulombic, van der Waals, and bonded energy terms. Additional energy terms can be added to Equation S1 to incorporate pressure, stress, 
electric fields, and other environmental variables. The potential energy for Coulombic interactions is given as:

$$
E_{\text {Coul }}=\frac{e^{2}}{4 \pi \epsilon_{\circ}} \sum_{i \neq j} \frac{q_{i} q_{j}}{r_{i j}}
$$

where $q_{i}$ and $q_{j}$ are the partial charges for atoms $i$ and $j$ separated by distance $r_{i j}$. The $E_{\mathrm{VDW}}$ term is often incorporated in Equation S1 as a L-J potential to represent short range interactions between atom pairs. It is presented in Equation $\mathrm{S} 3$ using $\varepsilon$ and $\sigma$ notation:

$$
E_{V D W}=\sum_{i \neq j} 4 \varepsilon_{i j}\left[\left(\frac{\sigma_{i j}}{r_{i j}}\right)^{12}-\left(\frac{\sigma_{i j}}{r_{i j}}\right)^{6}\right]
$$

where $\varepsilon_{i j}$ is the depth of the potential well and $\sigma_{i j}$ is the zero-crossing distance for the energy. Combining rules for the L-J parameters are used to mix nonequivalent (off-diagonal) atomic interactions thereby requiring reporting of only the single (diagonal) parameters (Table S1):

$$
\sigma_{i j}=\frac{\sigma_{i}+\sigma_{j}}{2} \quad \varepsilon_{i j}=\left(\varepsilon_{i} \varepsilon_{j}\right)^{1 / 2}
$$

Clayff implements a conventional geometric mean for the energy term $\varepsilon_{i j}$ and an arithmetic mean for the zero-crossing distance $\sigma_{i j}$. A Simple Point Charge (SPC) model ${ }^{2}$ is used for the L-J parameters of water molecules in Clayff. Improvements in modeling water polarization using the effective pair potential of the SPC/E water model $^{3}$ can be also used with Clayff potentials, however the SPC/E partial charges are not fully consistent with the charges associated with the framework oxygen atoms (see Table S1).

Table S2 presents the bonded parameters for Clayff potentials. A harmonic potential is used to describe the potential energy of -O-H bonds associated with water and hydroxyl groups:

$$
E_{\text {harmonic stretch }}=k_{1}\left(r-r_{\mathrm{o}}\right)^{2}
$$


where $r_{\mathrm{o}}$ is the equilibrium bond distance. Similarly, a harmonic function is used to describe the potential energy for the bond-angle bend for water molecules and for selected metal hydroxyl groups (edges and truncated surfaces):

$$
E_{\text {harmonic bend }}=k_{2}\left(\theta-\theta_{o}\right)^{2}
$$

where $\theta_{\mathrm{o}}$ represents the equilibrium bond angle for a three-body interaction. Full flexibility of water molecules is therefore incorporated in Clayff by use of harmonic bond stretch and bondangle bending terms. ${ }^{4}$ A Morse potential has been successfully used in Clayff simulations of clay minerals involving TOT layers with dioctahedral and trioctahedral structures:

$$
E_{\text {Morse stretch }}=D_{o}\left[1-e^{-\alpha\left(r-r_{o}\right)}\right]^{2}
$$

where $D_{o}$ is the dissociation energy, $r_{o}$ is the equilibrium bond distance, and $\alpha$ is an additional fitting parameter representing the width of the potential energy well. Use of a Morse potential for modeling hydroxyl groups has led to improved simulations of vibrational spectra for TOT clay minerals. ${ }^{5}$

Recognizing the challenge of modeling edge sites of layered phases and other complex materials, Clayff has been recently updated to accurately predict the librational motions of hydroxyl groups associated with edges and other truncated surfaces.$^{6-8}$ Harmonic bond-angle bend terms (Equation S6) were parameterized from results of DFT-MD simulations and were optimized to accurately predict structure and power spectra. Force constants and equilibrium bond angles are presented in Table S2 for selected metal atom types associated with protonated oxygen species. Note, these bonded M-O-H parameters replace the three-body parameters published in the original Clayff potential set, which were cast in a different functional form and difficult to implement in practice. They can be implemented as special nonbonded three-body terms (e.g., LAMMPS “pair style nb3b/harmonic”; Materials Studio Forcite "NON- 
BONDED_3-BODY”). These M-O-H parameters can also be implemented as angle terms without associated bond terms, thereby bypassing the standard 1-3 or 1-4 bond exclusion rules. However, in this case, the angle terms continue to be evaluated even when the hydroxyl group moves away from the metal (M) during the simulation.

Clayff potentials are typically assigned as atom types in molecule models involving simulation cells with periodic boundary conditions. Some graphical-based software packages, such as Materials Studio, require molecular models be created without bonds for the metaloxygen framework so that the nonbonded Clayff potentials are properly implemented. Water molecules and hydroxyl groups would require graphical bonds, and have corresponding bonded potentials (bond stretch and bond-angle bend) for evaluating their bond energy contributions. There are several pre- and post-processing software tools available to the user for preparing molecular models for Clayff-based simulations and for analyzing molecular structures from atomic trajectories obtained from MD simulations. ${ }^{9-11}$ Many of these tools are developed by academic institutions and are customized for specific applications. An open-source MATLAB library has been recently developed by Holmboe ${ }^{12}$ to create models of layered minerals and other phases, to assign Clayff attributes, to protonate and solvate models, and to analyze bonding and atomic structure for models from multiple simulations. 
Table S1. Nonbond Parameters for the Clayff Force Field

\begin{tabular}{|l|c|c|c|c|}
\hline \multicolumn{1}{|c|}{ species } & symbol & $\begin{array}{c}\text { charge } \\
(e)\end{array}$ & $\begin{array}{c}\varepsilon \\
(\mathrm{kcal} / \mathrm{mol})\end{array}$ & $\begin{array}{c}\sigma \\
(\AA)\end{array}$ \\
\hline SPC water hydrogen & $\mathrm{h}^{*}$ & 0.4100 & & \\
\hline SPC water oxygen & $\mathrm{o}^{*}$ & -0.8200 & 0.1554 & 3.1655 \\
\hline SPC/E water hydrogen & $\mathrm{h}^{*}$ & 0.4238 & & \\
\hline SPC/E water oxygen & $\mathrm{o}^{*}$ & -0.8476 & 0.1554 & 3.1655 \\
\hline hydroxyl hydrogen & ho & 0.4250 & & \\
\hline hydroxyl oxygen & oh & -0.9500 & 0.1554 & 3.1655 \\
\hline bridging oxygen & ob & -1.0500 & 0.1554 & 3.1655 \\
\hline $\begin{array}{l}\text { bridging oxygen with } \\
\text { octahedral substitution }\end{array}$ & obos & -1.1808 & 0.1554 & 3.1655 \\
\hline $\begin{array}{l}\text { bridging oxygen with } \\
\text { tetrahedral substitution }\end{array}$ & obts & -1.1688 & 0.1554 & 3.1655 \\
\hline $\begin{array}{l}\text { bridging oxygen with } \\
\text { double substitution }\end{array}$ & obss & -1.2996 & 0.1554 & 3.1655 \\
\hline $\begin{array}{l}\text { hydroxyl oxygen with } \\
\text { substitution }\end{array}$ & ohs & -1.0808 & 0.1554 & 3.1655 \\
\hline tetrahedral silicon & st & 2.1000 & $1.8405 \times 10^{-6}$ & 3.3020 \\
\hline octahedral aluminum & ao & 1.5750 & $1.3298 \times 10^{-6}$ & 4.2712 \\
\hline tetrahedral aluminum & at & 1.5750 & $1.8405 \times 10^{-6}$ & 3.3020 \\
\hline octahedral magnesium & $\mathrm{mgo}$ & 1.3600 & $9.0298 \times 10^{-7}$ & 5.2643 \\
\hline hydroxide magnesium & $\mathrm{mgh}$ & 1.0500 & $9.0298 \times 10^{-7}$ & 5.2643 \\
\hline octahedral calcium & cao & 1.3600 & $5.0298 \times 10^{-6}$ & 5.5667 \\
\hline hydroxide calcium & cah & 1.0500 & $5.0298 \times 10^{-6}$ & 5.5667 \\
\hline octahedral iron & feo & 1.5750 & $9.0298 \times 10^{-7}$ & 4.9062 \\
\hline octahedral lithium & lio & 0.5250 & $9.0298 \times 10^{-7}$ & 4.2101 \\
\hline aqueous sodium ion & $\mathrm{Na}$ & 1.0 & 0.1301 & 2.3500 \\
\hline aqueous potassium ion & $\mathrm{K}$ & 1.0 & 0.1000 & 3.3340 \\
\hline aqueous cesium ion & $\mathrm{Cs}$ & 1.0 & 0.1000 & 3.8310 \\
\hline aqueous calcium ion & $\mathrm{Ca}$ & 2.0 & 0.1000 & 2.8720 \\
\hline aqueous barium ion & $\mathrm{Ba}$ & 2.0 & 0.0470 & 3.8166 \\
\hline aqueous chloride ion & $\mathrm{Cl}$ & -1.0 & 0.1001 & 4.4000 \\
\hline
\end{tabular}


Table S2. Bond Parameters for the Clayff Force Field

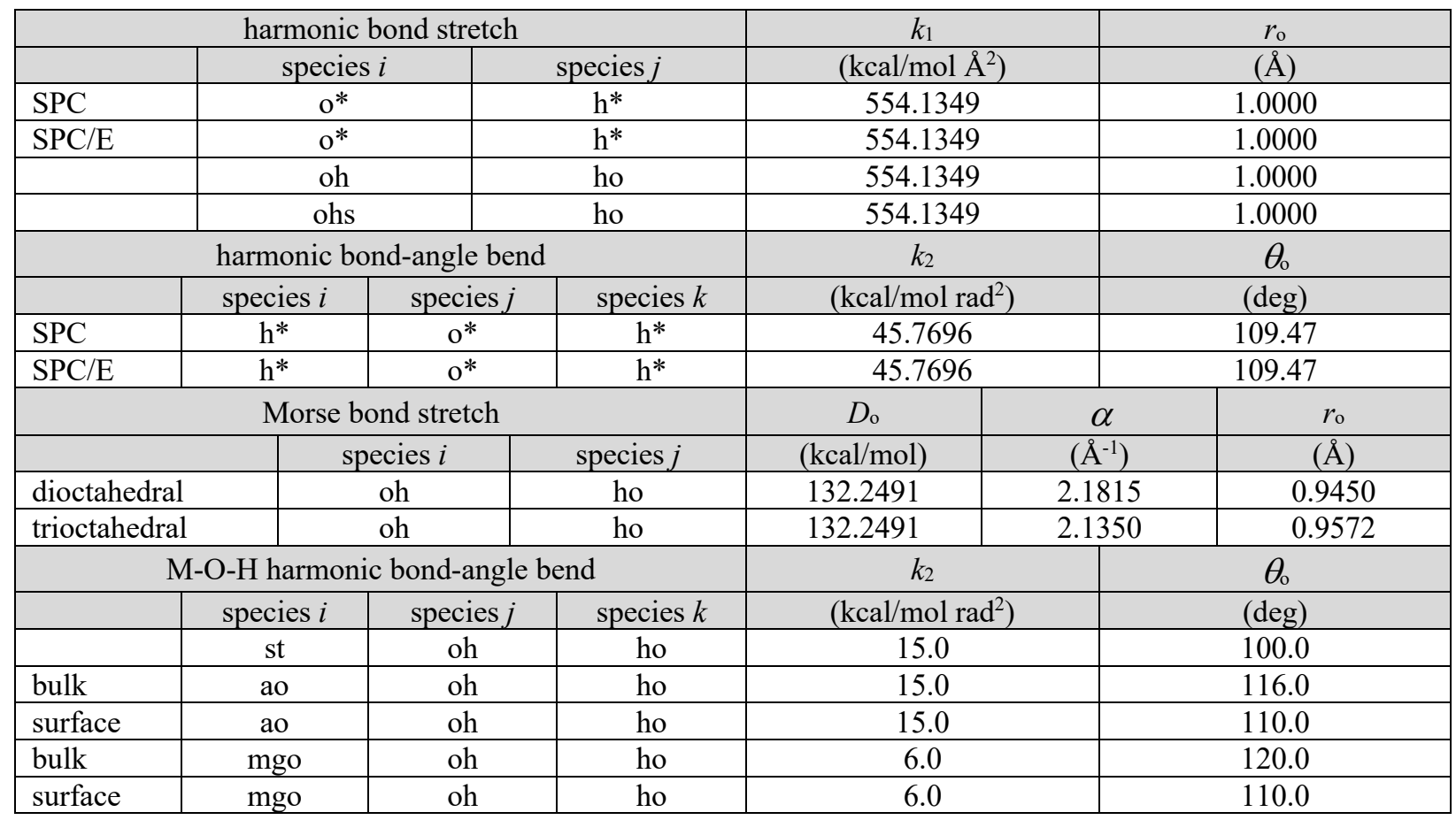

\section{SAMPLE CLAYFF INPUT FILES}

Several sample program input files incorporating Clayff parameters are provided below to assist the user in implementing Clayff in commonly-used classical mechanics and molecular simulation software packages. The software programs include LAMMPS, GROMACS, and Materials Studio modules Forcite and Discover. Please note the following:

- Other ion-water parameters ${ }^{13-16}$ in the LJ 6-12 format could be used in place of (or in addition to) the VDW parameters provided in the original Clayff set. Off-diagonal parameters for ionmineral interactions can then be derived using the appropriate mixing rules.

- Some software packages have options for automated assignment of atom types (partial charges, L-J parameters). These parameter assignments should always be checked since the algorithms may not be appropriate for all model systems. 
- There may be subtle differences in parameter values between the sample input files for these codes and those provided in Tables S1 and S2. Often such input files are recreated as restart files during program execution and often include extraneous significant digits for the Clayff parameters.

- Nothing is guaranteed in these examples, and the software user is reminded to consult the original publications (including this review article) and the software manuals to ensure that parameter choices and program options are appropriately implemented for the technical problem at hand.

\section{Clayff for LAMMPS}

\begin{tabular}{|c|c|c|c|c|}
\hline \multicolumn{5}{|c|}{ Pair Coeffs \# lj/cut/coul/long } \\
\hline 1 & 0.0000018402 & 3.3019566252 & \# st tetrahedral $\mathrm{Si}$ & 2.1 \\
\hline 2 & 0.0000013297 & 4.2713219316 & \# ao octahedral Al & 1.575 \\
\hline 3 & 0.0000018402 & 3.3019566252 & \# at tetrahedral Al & 1.575 \\
\hline 4 & 0.0000009030 & 5.2643258688 & \# mgo octahedral $\mathrm{Mg}$ & 1.36 \\
\hline 5 & 0.0000009030 & 5.2643258688 & \# mgh hydroxide $\mathrm{Mg}$ & 1.05 \\
\hline 6 & 0.0000050301 & 5.5666638636 & \# cao octahedral Ca & 1.36 \\
\hline 7 & 0.0000050219 & 5.5624343529 & \# cah hydroxide $\mathrm{Ca}$ & 1.05 \\
\hline 8 & 0.0000009030 & 4.9057651456 & \# feo octahedral $\mathrm{Fe}$ & 1.575 \\
\hline 9 & 0.0000009030 & 4.2105948665 & \# lio octahedral Li & 0.525 \\
\hline 10 & 0.1554164124 & 3.1655200879 & \# ob bridging oxygen & -1.05 \\
\hline 11 & 0.1554164124 & 3.1655200879 & \# obss oxygen double sub. & -1.2996 \\
\hline 12 & 0.1554164124 & 3.1655200879 & \# obts oxygen tet. sub. & -1.1688 \\
\hline 13 & 0.1554164124 & 3.1655200879 & \# obos oxygen oct. sub. & -1.1808 \\
\hline 14 & 0.1554164124 & 3.1655200879 & \# ohs hydroxyl o sub. & -1.0808 \\
\hline 15 & 0.1554164124 & 3.1655200879 & \# oh hydroxyl o & -0.95 \\
\hline 16 & 0.1554164124 & 3.1655200879 & \# o* spc water 0 & -0.82 \\
\hline 17 & 0.0000000000 & 0.0000000000 & \# ho hydroxyl H & 0.425 \\
\hline 18 & 0.0000000000 & 0.0000000000 & $\# \mathrm{~h} * \mathrm{spc}$ water $\mathrm{H}$ & 0.41 \\
\hline 19 & 0.1300999871 & 2.3500126639 & \# $\mathrm{Na}$ sodium ion & 1.0 \\
\hline 20 & 0.1000015417 & 3.3340059879 & \# $\mathrm{K}$ potassium ion & 1.0 \\
\hline 21 & 0.1000007161 & 3.8310403819 & \# Cs cesium ion & 1.0 \\
\hline 22 & 0.0999999980 & 2.8719902024 & \# Ca calcium ion & 2.0 \\
\hline 23 & 0.0470957194 & 3.8166120025 & \# Ba barium ion & 2.0 \\
\hline 27 & 0.1000998933 & 4.3999709805 & \# Cl chloride ion & -1.0 \\
\hline
\end{tabular}

Bond Coeffs \# harmonic

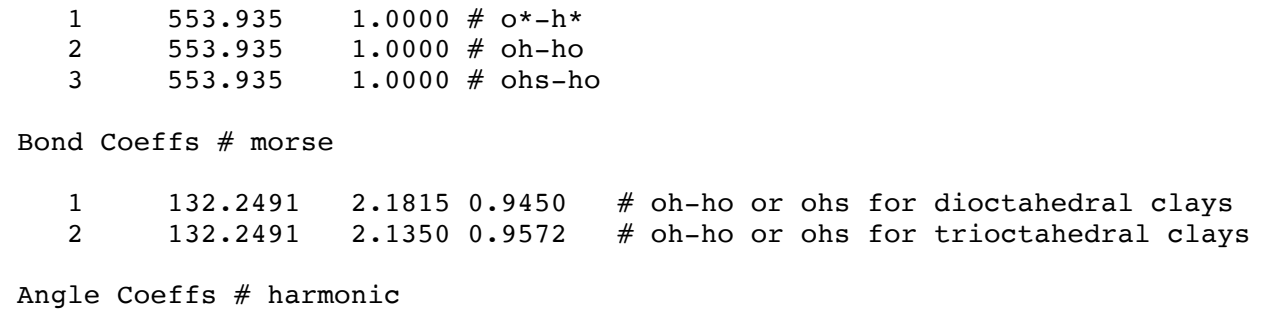




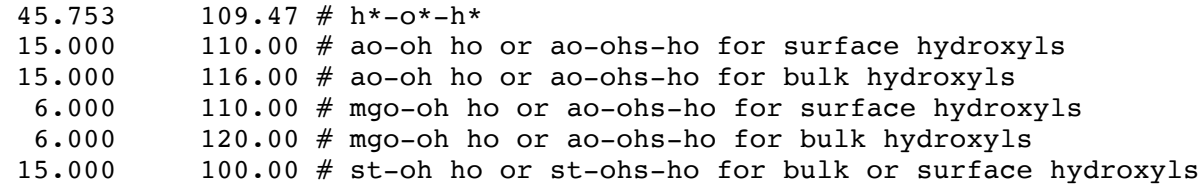

\section{Clayff for Materials Studio Forcite}

\begin{tabular}{|c|c|}
\hline \multicolumn{2}{|l|}{ VERSION 3.1 .02} \\
\hline \multicolumn{2}{|l|}{ END } \\
\hline \multicolumn{2}{|l|}{ \# } \\
\hline \multicolumn{2}{|l|}{ HEADER } \\
\hline \multicolumn{2}{|l|}{ END } \\
\hline \multicolumn{2}{|l|}{ \# } \\
\hline \multicolumn{2}{|l|}{ PREFERENCES } \\
\hline BONDS & $\mathrm{T}$ \\
\hline ANGLES & $\mathrm{T}$ \\
\hline COULOMB & $\mathrm{T}$ \\
\hline INVERSIONS & $\mathrm{F}$ \\
\hline TORSIONS & $\mathrm{T}$ \\
\hline UREY_BRADLEY & $\mathrm{F}$ \\
\hline STRETCH_STRETCH & $\mathrm{F}$ \\
\hline SEPARATED_STRETCH_STRETCH & $\mathrm{F}$ \\
\hline STRETCH_BEND_STRETCH & $\mathrm{F}$ \\
\hline BEND_BEN̄D & $\mathrm{F}$ \\
\hline TORSION_STRETCH & $\mathrm{F}$ \\
\hline TORSION_BEND_BEND & $\mathrm{F}$ \\
\hline BEND_TORSION_BEND & $\mathrm{F}$ \\
\hline STRETCH_TORSION_STRETCH & $\mathrm{F}$ \\
\hline HYDROGEN_BONDS ${ }^{-}$ & $\mathrm{F}$ \\
\hline DIAGONAL_VAN_DER_WAALS & $\mathrm{T}$ \\
\hline OFF_DIAGONAL_VAN_DER_WAALS & $\mathrm{T}$ \\
\hline IGNORE_UNDEF INNED_TERM $\bar{M}$ & $\mathrm{~T}$ \\
\hline NON-BONDED_3-BODY & $\mathrm{F}$ \\
\hline SHRINK_CH_B্BONDS & $\mathrm{F}$ \\
\hline SHRINK_CH_H_ATOM & $\mathrm{H} \_\mathrm{C}$ \\
\hline SHRINK_CH_FĀCTOR & 0.91500 \\
\hline SINGLE_TORSION & $\mathrm{F}$ \\
\hline SCALE_TORSIONS_ABOUT_COMMON_BOND & $\mathrm{F}$ \\
\hline SCALE_BY_N_DEFINAED_TORSIONS ${ }^{-}$ & $\mathrm{T}$ \\
\hline EXOCYCLIC_TORSIONS_SCALE_FACTOR & 0.40000 \\
\hline SINGLE_INVERSION & $\mathrm{F}$ \\
\hline H-BOND_METHOD & SPLINE \\
\hline H-BOND_LIST & $\mathrm{T}$ \\
\hline H-BOND_DIRECT_RCUT & 4.00000 \\
\hline H-BOND_SPLINE_DISTANCE_ON & 4.00000 \\
\hline H-BOND_SPLINE_DISTANCE_OFF & 4.50000 \\
\hline H-BOND_SPLINE_ANGLE_ON & 65.00000 \\
\hline H-BOND_SPLINE_ANGLE_OFF & 75.00000 \\
\hline H-BOND_LIST_DI STANCE_OFF & 6.50000 \\
\hline H-BOND_LIST_ANGLE_OFF & 90.00000 \\
\hline NB_NEIGHBOUR_SEARCH_METHOD & LIST \\
\hline NON & 2.00000 \\
\hline H-BŌND_BŪFFER_DI STANCE & 2.00000 \\
\hline COU_DIĒLETRIC_CONSTANT & 1.00000000000000000 \\
\hline COU_INTER_CUT_OFF & 8.00000 \\
\hline COU_SPLINE_OFF & 8.50000 \\
\hline COU_SPLINE_ON & 8.00000 \\
\hline 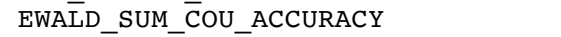 & 0.00100 \\
\hline EWALD_SUM_COU_ETA & 3.20600 \\
\hline EWALD_SUM_COU_KCUT & 0.40900 \\
\hline EWALD_SUM_COU_RCUT & 10.85000 \\
\hline EWALD_SUM_COU_OPTIMIZE & SMART \\
\hline 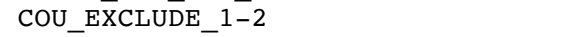 & $\mathrm{T}$ \\
\hline COU_EXCLUDE_1-3 & $\mathrm{T}$ \\
\hline COU_EXCLUDE_1-4 & $\mathrm{F}$ \\
\hline COU_1-4_SCALEE_FACTOR & 1.00000 \\
\hline
\end{tabular}




\begin{tabular}{|c|c|c|c|c|c|c|c|c|}
\hline & \multicolumn{5}{|c|}{ EWALD } \\
\hline \multicolumn{4}{|c|}{ COU_DIRECT_CUT-OFF } & \multicolumn{5}{|c|}{8.00000} \\
\hline \multicolumn{4}{|c|}{ VDW_COMBINĀTION_RULE } & \multicolumn{5}{|c|}{ ARITHMETIC } \\
\hline \multicolumn{4}{|c|}{ VDW_INTER_CUT_OFF } & \multicolumn{5}{|c|}{$\begin{array}{r}\text { ARITHMETIC } \\
8.00000\end{array}$} \\
\hline VDW_E & $E \_1-2$ & & & $\mathrm{~T}$ & & & & \\
\hline VDW_E & E_1-3 & & & T & & & & \\
\hline VDW_E & E_1-4 & & & $\mathrm{F}$ & & & & \\
\hline VDW_1 & $A \bar{L} E \_F$ & ETOR & & & & $000 c$ & & \\
\hline VDW_M & & & & & LIN & & & \\
\hline VDW_S & ON & & & & & 000 & & \\
\hline VDW_S & $\mathrm{OFF}$ & & & & & 0000 & & \\
\hline EWALD & VDW_O & IMIZE & & & ART & & & \\
\hline EWALD & VDW_A & UURACY & & & & 010 & & \\
\hline EWALD & VDW_E & & & & & 8000 & & \\
\hline EWALD & VDW_K & & & & & 6000 & & \\
\hline EWALD & VDW_R & & & & & 000 & & \\
\hline EWALD & VDW_R & CCUT & & & & 000 & & \\
\hline FAST_ & SUM & ATIO & & & 10 . & 000 & & \\
\hline SLOW_ & SUM & ATIO & & & & 000 & & \\
\hline MINIM & $\bar{A} G E$ & & & $\mathrm{~F}$ & & & & \\
\hline ASSIG & & & & $\mathrm{F}$ & & & & \\
\hline ASSIG & RGE & & & $\mathrm{F}$ & & & & \\
\hline ASSIG & RIDIZ & IION & & $\mathrm{F}$ & & & & \\
\hline ASSIG & BOND & SNTER & & $\mathrm{F}$ & & & & \\
\hline ATOM & & & & $\mathrm{F}$ & & & & \\
\hline ATOM & ALL & & & $\mathrm{T}$ & & & & \\
\hline CALCU & BOND_ & RDER & & $\mathrm{F}$ & & & & \\
\hline END & & & & & & & & \\
\hline \# & & & & & & & & \\
\hline АтОМту & & & & & & & & \\
\hline $\mathrm{h} *$ & $\mathrm{H}$ & 1.00790 & 0.4100 & 1 & 0 & 0 & & spc water $\mathrm{H}$ \\
\hline ho & $\mathrm{H}$ & 1.00794 & 0.4250 & 1 & 0 & 0 & $!$ & hydroxyl H \\
\hline o* & 0 & 15.99940 & -0.8200 & 3 & 0 & 0 & $!$ & spc water 0 \\
\hline oh & 0 & 15.99940 & -0.9500 & 3 & 0 & 0 & $! 1$ & hydroxyl o \\
\hline$o b$ & 0 & 15.99940 & -1.0500 & 3 & 0 & 0 & ! 1 & bridging oxygen \\
\hline st & $\mathrm{Si}$ & 28.08550 & 2.1000 & 3 & 0 & 0 & $!$ & tetrahedral Si \\
\hline ao & Al & 26.98154 & 1.5750 & 6 & 0 & 0 & $!$ & octahedral Al \\
\hline at & Al & 26.98154 & 1.5750 & 3 & 0 & 0 & $!$ & tetrahedral Al \\
\hline mgo & $\mathrm{Mg}$ & 24.30500 & 1.3600 & 3 & 0 & 0 & $!$ & octahedral Mg \\
\hline cao & $\mathrm{Ca}$ & 40.08000 & 1.3600 & 6 & 0 & 0 & $!$ & octahedral $\mathrm{Ca}$ \\
\hline feo & $\mathrm{Fe}$ & 55.84700 & 1.5750 & 6 & 0 & 0 & $!$ & octahedral $\mathrm{Fe}$ \\
\hline lio & $\mathrm{Li}$ & 6.94000 & 0.5250 & 0 & 0 & 0 & $!$ & octahedral Li \\
\hline obss & 0 & 15.99940 & -1.2996 & 2 & 0 & 0 & $!$ & oxygen double sub. \\
\hline obts & 0 & 15.99940 & -1.1688 & 2 & 0 & 0 & $!$ & oxygen tet. sub. \\
\hline obos & 0 & 15.99940 & -1.1808 & 2 & 0 & 0 & $!$ & oxygen oct. sub. \\
\hline ohs & 0 & 15.99940 & -1.0808 & 2 & 0 & 0 & $!$ & hydroxyl o sub. \\
\hline $\mathrm{cah}$ & $\mathrm{Ca}$ & 40.08000 & 1.0500 & 6 & 0 & 0 & ! 1 & hydroxide $\mathrm{Ca}$ \\
\hline $\mathrm{mgh}$ & Mg & 24.30500 & 1.0500 & 3 & 0 & 0 & ! 1 & hydroxide Mg \\
\hline $\mathrm{Na}$ & $\mathrm{Na}$ & 22.98977 & 1.0000 & 7 & 0 & 0 & $!$ & sodium ion \\
\hline $\mathrm{K}$ & $\mathrm{K}$ & 39.09830 & 1.0000 & 0 & 0 & 0 & $! 1$ & potassium ion \\
\hline Cs & $\mathrm{Cs}$ & 132.90546 & 1.0000 & 0 & 0 & 0 & ! & cesium ion \\
\hline $\mathrm{Ca}$ & $\mathrm{Ca}$ & 40.08000 & 2.0000 & 6 & 0 & 0 & $!$ & calcium ion \\
\hline $\mathrm{Ba}$ & $\mathrm{Ba}$ & 137.33000 & 2.0000 & 7 & 0 & 0 & ! 1 & barium ion \\
\hline $\mathrm{Cl}$ & $\mathrm{Cl}$ & 35.45270 & -1.0000 & 0 & 0 & 0 & $!$ & chloride ion \\
\hline END & & & & & & & & \\
\hline \# & & & & & & & & \\
\hline DIAGON & & & & & & & & \\
\hline $\mathrm{h} *$ & IGN & & 4.5774998 & 648 & 596 & & & \\
\hline ho & IGN & & 4.5774998 & 648 & 596 & & & \\
\hline o* & LJ & 12 & 3.5532000 & 648 & 985 & & & 0.1553999930620193 \\
\hline oh & $\mathrm{LJ}_{-}$ & 12 & 3.5532000 & & 985 & & & 0.1553999930620193 \\
\hline$o b$ & $\mathrm{LJ}_{-}$ & 12 & 3.5532000 & & 985 & & & 0.1553999930620193 \\
\hline st & $\mathrm{LJ}_{-}^{-}$ & 12 & $3.706350 c$ & & 507 & & & $0.1840499976424326 \mathrm{E}-05$ \\
\hline ao & $\mathrm{LJ}$ & 12 & 4.7943201 & & 348 & & & $0.1329800056737440 \mathrm{E}-05$ \\
\hline at & $\mathrm{LJ}_{-}^{-}$ & 12 & $3.706350 c$ & & 507 & & & $0.1840499976424326 \mathrm{E}-05$ \\
\hline mgo & $\mathrm{LJ}_{-}$ & 12 & 5.9090161 & & 736 & & & $0.9029800480675476 \mathrm{E}-06$ \\
\hline cao & $\mathrm{LJ}_{-}$ & 12 & 6.2484030 & & 178 & & & $0.5029799922340317 \mathrm{E}-05$ \\
\hline feo & $\mathrm{LJ}_{-}$ & 12 & 5.5070118 & & 377 & & & $0.9029800480675476 \mathrm{E}-06$ \\
\hline lio & $\mathrm{LJ}_{-}$ & 12 & $4.725677 \mathrm{C}$ & & 217 & & & $0.9029799912241288 \mathrm{E}-06$ \\
\hline obss & $\mathrm{LJ}_{-}^{-}$ & 12 & 3.5532000 & & 985 & & & 0.1553999930620193 \\
\hline obts & $\mathrm{LJ}_{-}$ & 12 & 3.5532000 & & 985 & & & 0.1553999930620193 \\
\hline obos & $\mathrm{LJ}_{-}^{-}$ & 12 & 3.5532000 & & 985 & & & 0.1553999930620193 \\
\hline ohs & $\mathrm{LJ}_{-}^{-}$ & 12 & 3.5532000 & 648 & 985 & & & 0.1553999930620193 \\
\hline
\end{tabular}




\begin{tabular}{|c|c|c|c|c|c|}
\hline $\mathrm{cah}$ & LJ_6_12 & \multicolumn{2}{|c|}{6.242803096771240} & \multicolumn{2}{|c|}{$0.5029799922340317 \mathrm{E}-05$} \\
\hline $\mathrm{mgh}$ & $\mathrm{LJ}^{-} 6^{-} 12$ & \multicolumn{2}{|c|}{5.909016132354736} & \multicolumn{2}{|c|}{$0.9029799912241288 \mathrm{E}-06$} \\
\hline $\mathrm{Na}$ & LJ_6_12 & \multicolumn{2}{|c|}{2.637800011444092} & \multicolumn{2}{|c|}{0.1300999969244003} \\
\hline $\mathrm{K}$ & LJ_6_12 & 3.742 & 00000000000 & 0.10 & 00000 \\
\hline $\mathrm{Cs}$ & LJ_6_12 & 4.300 & 00000000000 & 0.10 & 00000 \\
\hline $\mathrm{Ca}$ & LJ_6_12 & 3.223 & 00000000000 & 0.10 & 00000 \\
\hline $\mathrm{Ba}$ & LJ_6_12 & 4.284 & 00000000000 & 0.04 & 00000 \\
\hline $\mathrm{Cl}$ & LJ_6_-12 & 4.938 & 00000000000 & 0.10 & 92772 \\
\hline END & & & & & \\
\hline \# & & & & & \\
\hline ATOM_' & RULES & & & & \\
\hline $\mathrm{h} *$ & $\mathrm{H}$ & 0 & 0 & 1 & 1 \\
\hline & 0 & 1 & 0 & 1 & 1 \\
\hline & $\mathrm{H}$ & 0 & 0 & 1 & 1 \\
\hline & ** & 0 & 0 & 0 & -1 \\
\hline o* & 0 & 0 & 0 & 2 & 1 \\
\hline & $\mathrm{H}$ & 0 & 0 & 0 & 1 \\
\hline & $\mathrm{H}$ & 0 & 0 & 0 & 1 \\
\hline $\mathrm{ob}$ & $\mathrm{o}$ & 0 & 0 & 1 & 1 \\
\hline & $\mathrm{H}$ & 0 & 0 & 0 & -1 \\
\hline st & $\mathrm{Si}$ & 3 & 0 & 1 & 1 \\
\hline & 0 & 0 & 0 & 0 & 1 \\
\hline ao & Al & 6 & 0 & 1 & 1 \\
\hline & 0 & 0 & 0 & 0 & 1 \\
\hline at & Al & 3 & 0 & 1 & 1 \\
\hline & 0 & 0 & 0 & 0 & 1 \\
\hline $\mathrm{Na}$ & $\mathrm{Na}$ & 0 & 0 & 0 & 1 \\
\hline $\mathrm{K}$ & $\mathrm{K}$ & 0 & 0 & 0 & 1 \\
\hline Cs & Cs & 0 & 0 & 0 & 1 \\
\hline $\mathrm{Ca}$ & $\mathrm{Ca}$ & 0 & 0 & 0 & 1 \\
\hline $\mathrm{Ba}$ & $\mathrm{Ba}$ & 0 & 0 & 0 & 1 \\
\hline $\mathrm{Cl}$ & $\mathrm{Cl}$ & 0 & 0 & 0 & 1 \\
\hline $\mathrm{Mg}$ & $\mathrm{Mg}$ & 0 & 0 & 0 & 1 \\
\hline mgo & Mg & 0 & 0 & 0 & 1 \\
\hline cáo & $\mathrm{Ca}$ & 0 & 0 & 0 & 1 \\
\hline feo & $\mathrm{Fe}$ & 6 & 0 & 0 & 1 \\
\hline lio & $\mathrm{Li}$ & 6 & 0 & 0 & 1 \\
\hline oh & 0 & 0 & 0 & 3 & 1 \\
\hline & $\mathrm{H}$ & 0 & 0 & 0 & 1 \\
\hline & $* *$ & 6 & 0 & 0 & 1 \\
\hline & $* *$ & 6 & 0 & 0 & 1 \\
\hline ho & $\mathrm{H}$ & 0 & 0 & 1 & 1 \\
\hline & 0 & 0 & 0 & 1 & 1 \\
\hline & ** & 6 & 0 & 0 & 1 \\
\hline ohs & 0 & 0 & 0 & 2 & 1 \\
\hline & $\mathrm{H}$ & 0 & 0 & 0 & 1 \\
\hline & $\mathrm{Mg}$ & 6 & 0 & 0 & 1 \\
\hline obts & 0 & 0 & 0 & 2 & 1 \\
\hline & Al & 3 & 0 & 0 & 1 \\
\hline & ** & 0 & 0 & 0 & 1 \\
\hline obos & 0 & 0 & 0 & 2 & 1 \\
\hline & Al & 6 & 0 & 0 & 1 \\
\hline & $\mathrm{Mg}$ & 6 & 0 & 0 & 1 \\
\hline obss & 0 & 0 & 0 & 3 & 1 \\
\hline & Al & 3 & 0 & 0 & 1 \\
\hline & $\mathrm{Mg}$ & 6 & 0 & 0 & 1 \\
\hline & $* *$ & 6 & 0 & 0 & 1 \\
\hline cah & $\mathrm{Ca}$ & 6 & 0 & 1 & 1 \\
\hline & 0 & 0 & 0 & 1 & 1 \\
\hline & $\mathrm{Ca}$ & 6 & 0 & 0 & 1 \\
\hline $\mathrm{mgh}$ & $\mathrm{Mg}$ & 6 & 0 & 1 & 1 \\
\hline & 0 & 0 & 0 & 1 & 1 \\
\hline & $\mathrm{Mg}$ & 6 & 0 & 0 & 1 \\
\hline END & & & & & \\
\hline \# & & & & & \\
\hline \# & & & & & \\
\hline OFF_D & _VDW & & & & \\
\hline END & & & & & \\
\hline$\#$ & & & & & \\
\hline BOND_ & & & & & \\
\hline $0 *^{-}$ & & HARMONIC & 1108.2697 & 1.0000 & \\
\hline oh & & HARMONIC & 1108.2697 & 1.0000 & \\
\hline ohs & & HARMONIC & 1108.2697 & 1.0000 & \\
\hline
\end{tabular}




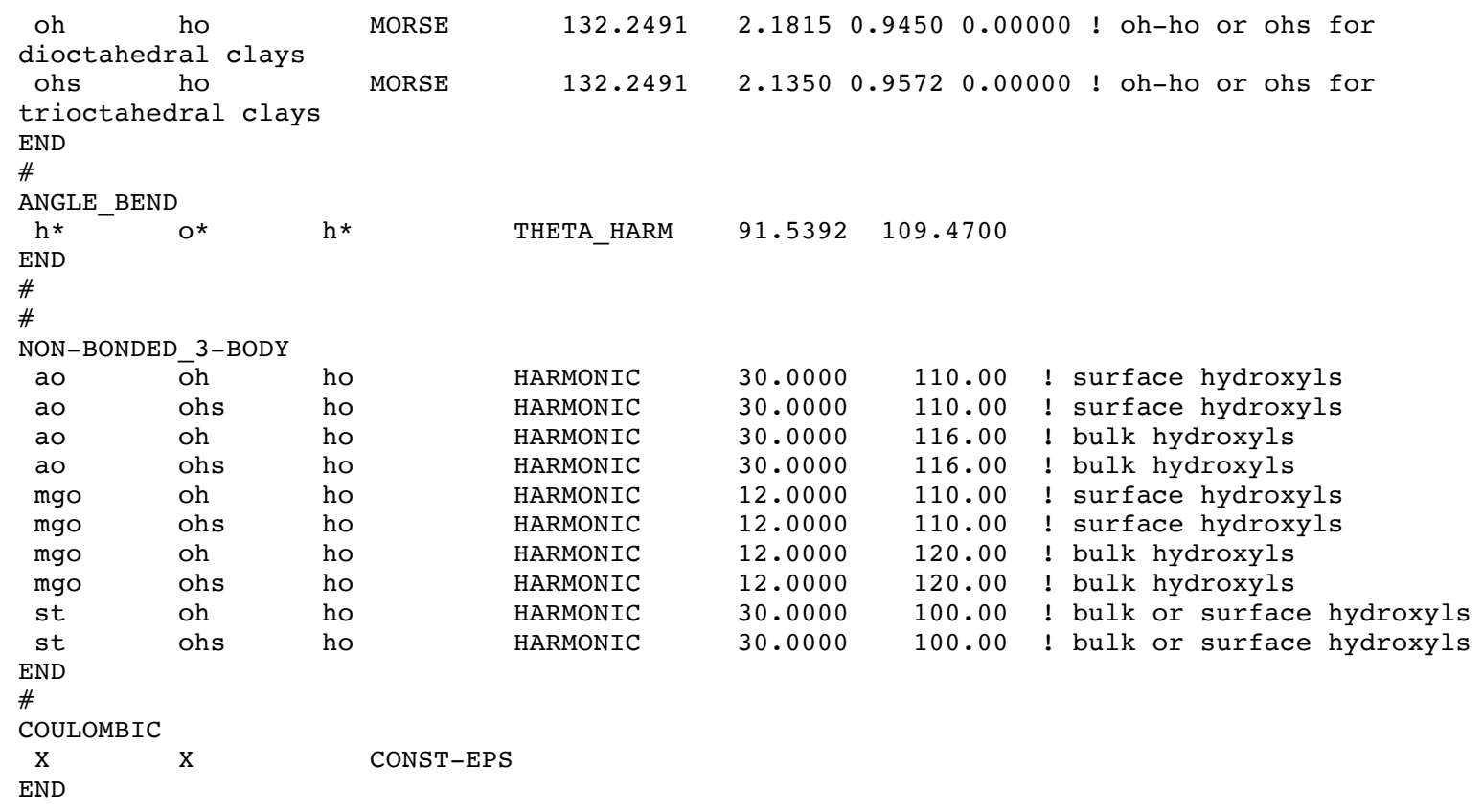

\section{Clayff for Materials Studio Discover}

! Discover .frc

\#atom_types

$\begin{array}{lcccc}\text { !Ver Ref } & \text { Type } & \text { Mass } & \text { Element } \\ \text { !--- --- } & --- & ------ & -----\end{array}$

Connections Description and charge

$1.0=-\quad$ st $\quad 28.08550$

$1.0 \quad-$ ao 26.98154

$1.0--$ at 26.98154

1.0 -- mgo 24.30500

1.0 -- mgh 24.30500

1.0 -- cao 40.08000

1.0 -- cah 40.08000

1.0 -- feo 55.84700

1.0 -- lio 6.941000

$1.0 \quad--$ ob 15.99940

1.0 -- obss 15.99940

1.0 -- obts 15.99940

1.0 -- obos 15.99940

1.0 -- ohs 15.99940

1.0 -- oh 15.99940

$1.0 \quad--\quad$ o* $^{*} 15.99940$

$1.0 \quad-$ ho 1.007970

$1.0 \quad--\quad h * \quad 1.007970$

$1.0 \quad-\quad \mathrm{Na} \quad 22.99000$

$1.0 \quad-\quad$ K 39.10

$1.0 \quad--\quad$ Cs 132.9100

$1.0 \quad-\quad \mathrm{Ca} \quad 40.07980$

$1.0 \quad--\quad \mathrm{Ba} \quad 137.3300$

$1.0 \quad--\quad \mathrm{Cl} \quad 35.45300$

$\mathrm{Si}$
$\mathrm{Al}$
$\mathrm{Al}$
$\mathrm{Mg}$
$\mathrm{Mg}$
$\mathrm{Ca}$
$\mathrm{Ca}$
$\mathrm{Fe}$
$\mathrm{Li}$
$\mathrm{O}$
$\mathrm{O}$
$\mathrm{O}$
$\mathrm{O}$
$\mathrm{O}$
$\mathrm{O}$
$\mathrm{O}$
$\mathrm{H}$
$\mathrm{H}$
$\mathrm{Na}$
$\mathrm{K}$
$\mathrm{Cs}$
$\mathrm{Ca}$
$\mathrm{Ba}$
$\mathrm{Cl}$

4 tetrahedral silicon 2.1

octahedral aluminum 1.575

tetrahedral aluminum 1.575

octahedral magnesium 1.36

hydroxide magnesium 1.05

octahedral calcium 1.36

hydroxide calcium 1.05

octahedral iron $\quad 1.575$

octahedral lithium 0.525

bridging oxygen $\quad-1.05$

oxygen double sub. -1.2996

oxygen tet. sub. -1.1688

oxygen oct. sub. -1.1808

hydroxyl 0 sub. $\quad-1.0808$

hydroxyl $0 \quad-0.95$

spc water $0 \quad-0.82$

hydroxyl $\mathrm{H} \quad 0.425$

spc water $\mathrm{H} \quad 0.41$

sodium ion 1.0

potassium ion $\quad 1.0$

cesium ion 1.0

calcium ion 2.0

barium ion 2.0

chloride ion $\quad-1.0$

\#quadratic_bond

$\begin{array}{cccccc}>\mathrm{E}=\mathrm{K} 2 * & (\mathrm{R}-\mathrm{R} 0)^{\wedge} 2 & & \\ & & & & \\ \text { !Ver } & \mathrm{Ref} & \mathrm{I} & \mathrm{J} & \mathrm{R} 0 & \mathrm{~K} 2 \\ \text { !--- --- } & ---- & ---- & ---- & ------ \\ 1.0 & -- & \text { O* } & \mathrm{h} * & 1.0000 & 553.9350 \\ 1.0 & -- & \text { oh } & \text { ho } & 1.0000 & 553.9350\end{array}$




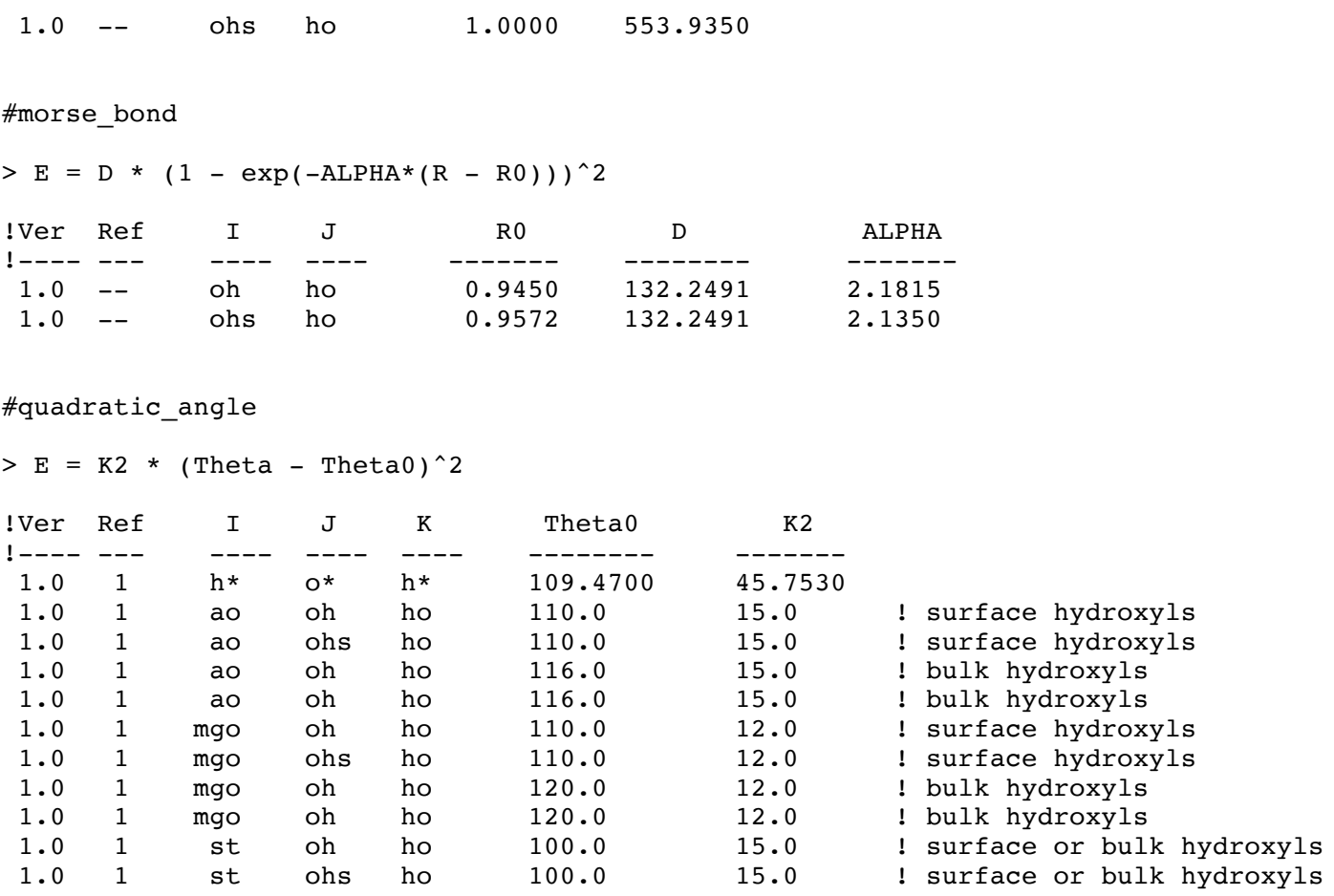

\#nonbond (12-6)

atype A-B

@combination geometric

$>\mathrm{E}=\mathrm{Aij} / \mathrm{r}^{\wedge} 12-\mathrm{Bij} / \mathrm{r}^{\wedge} 6$

$>$ where $A i j=\operatorname{sqrt}(A i * A j)$

$>\quad B i j=\operatorname{sqrt}(B i * B j)$

\begin{tabular}{|c|c|c|c|c|}
\hline !Ver & $\operatorname{Ref}$ & I & A & B \\
\hline$!----$ & --- & ---- & -------- & \\
\hline 1.0 & 1 & st & 12.3645 & 0.00954 \\
\hline 1.0 & 1 & ao & 196.1446 & 0.03230 \\
\hline 1.0 & 1 & at & 12.3645 & 0.00954 \\
\hline 1.0 & 1 & mgo & 1636.3265 & 0.07688 \\
\hline 1.0 & 1 & $\mathrm{mgh}$ & 1636.3265 & 0.07688 \\
\hline 1.0 & 1 & cao & 17814.73 & 0.5987 \\
\hline 1.0 & 1 & cah & 17624.076 & 0.595 \\
\hline 1.0 & 1 & feo & 702.54 & 0.0504 \\
\hline 1.0 & 1 & lio & 112.01 & 0.0201 \\
\hline 1.0 & 1 & $\mathrm{ob}$ & 629358.0000 & 625.50000 \\
\hline 1.0 & 1 & obss & 629358.0000 & 625.50000 \\
\hline 1.0 & 1 & obts & 629358.0000 & 625.50000 \\
\hline 1.0 & 1 & obos & 629358.0000 & 625.50000 \\
\hline 1.0 & 1 & ohs & 629358.0000 & 625.50000 \\
\hline 1.0 & 1 & oh & 629358.0000 & 625.50000 \\
\hline 1.0 & 1 & o* & 629358.0000 & 625.50000 \\
\hline 1.0 & 1 & ho & 0.00000001 & 0.00000 \\
\hline 1.0 & 1 & $\mathrm{~h}$ * & 0.00000001 & 0.00000 \\
\hline 1.0 & 1 & $\mathrm{Na}$ & 14763.1719 & 87.65132 \\
\hline 1.0 & 1 & $\mathrm{~K}$ & 754506.86 & 549.37 \\
\hline 1.0 & 1 & $\mathrm{Cs}$ & 3998193.96 & 1264.63 \\
\hline 1.0 & 1 & $\mathrm{Ca}$ & 125966.6068 & 224.46969 \\
\hline 1.0 & 1 & $\mathrm{Ba}$ & 1799606.56 & 582.25 \\
\hline 1.0 & 1 & $\mathrm{Cl}$ & 21081006.97 & 2905.31 \\
\hline
\end{tabular}




\section{Clayff for GROMACS}

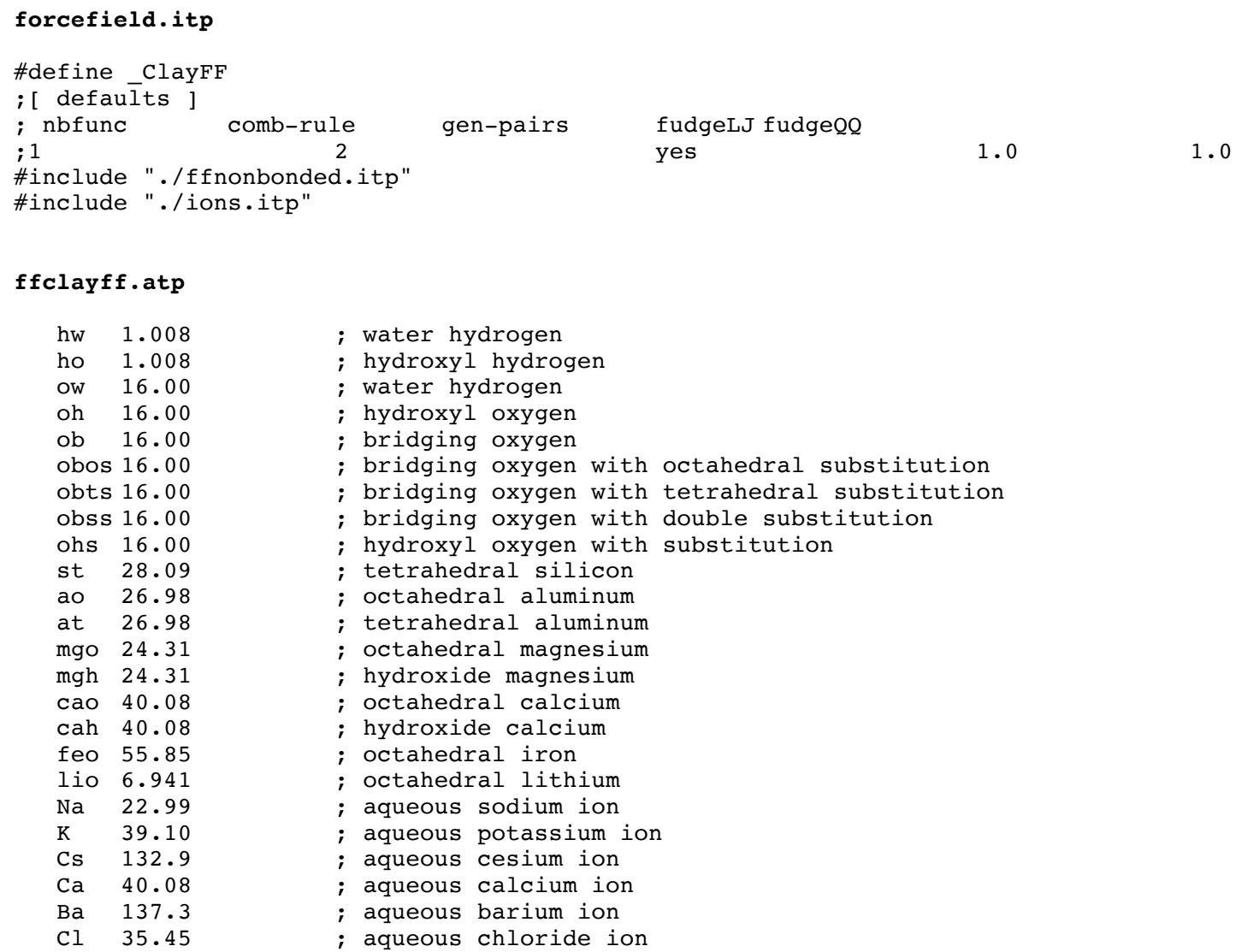

\section{ffnonbonded.itp}

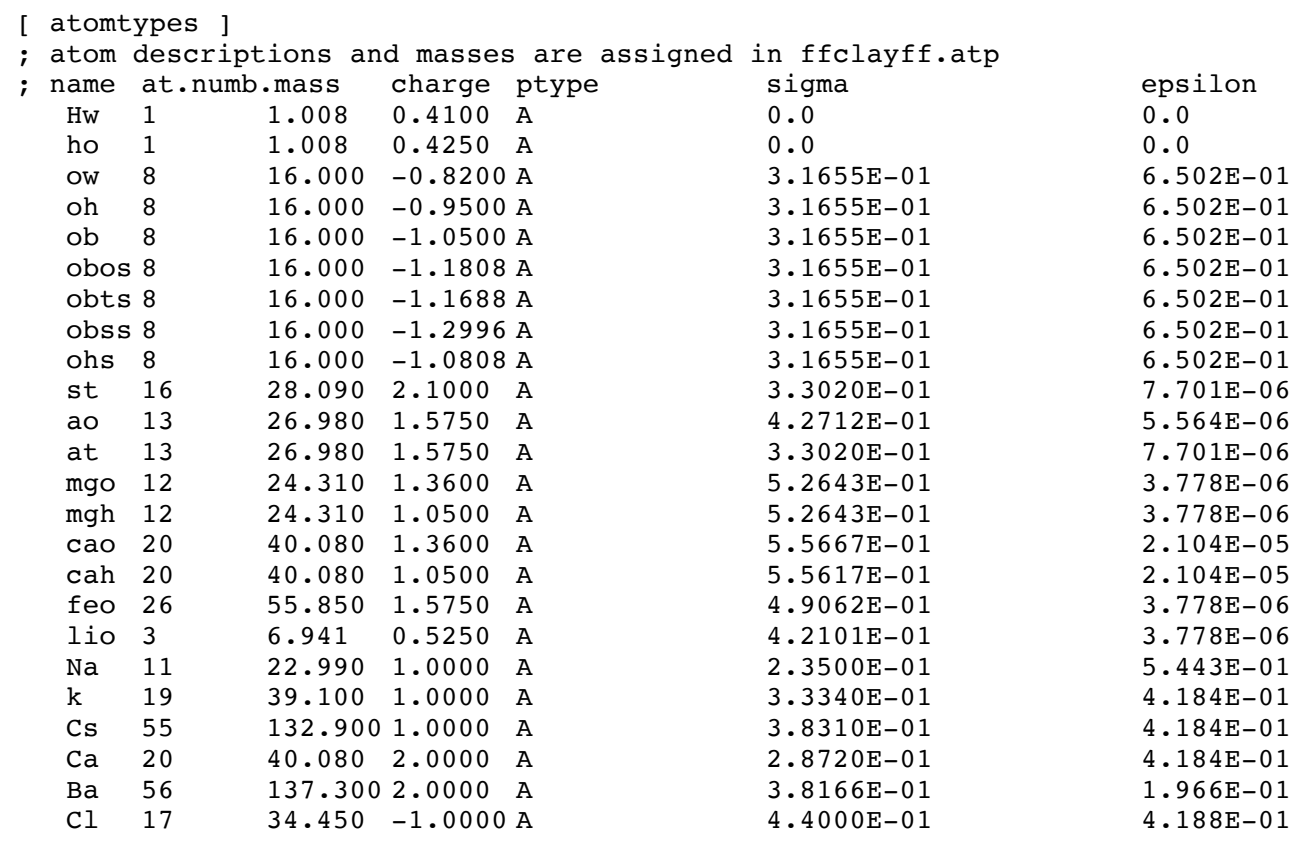




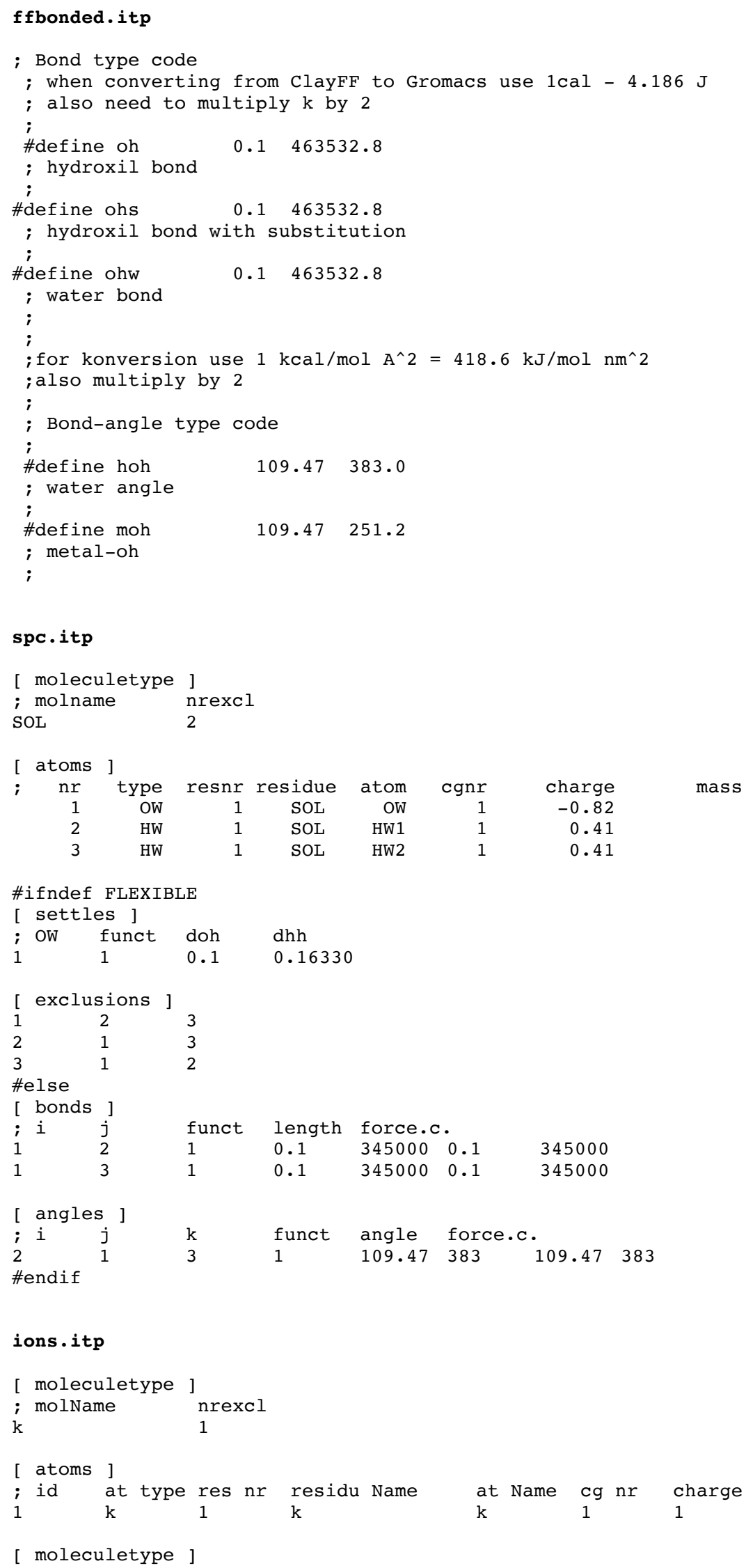




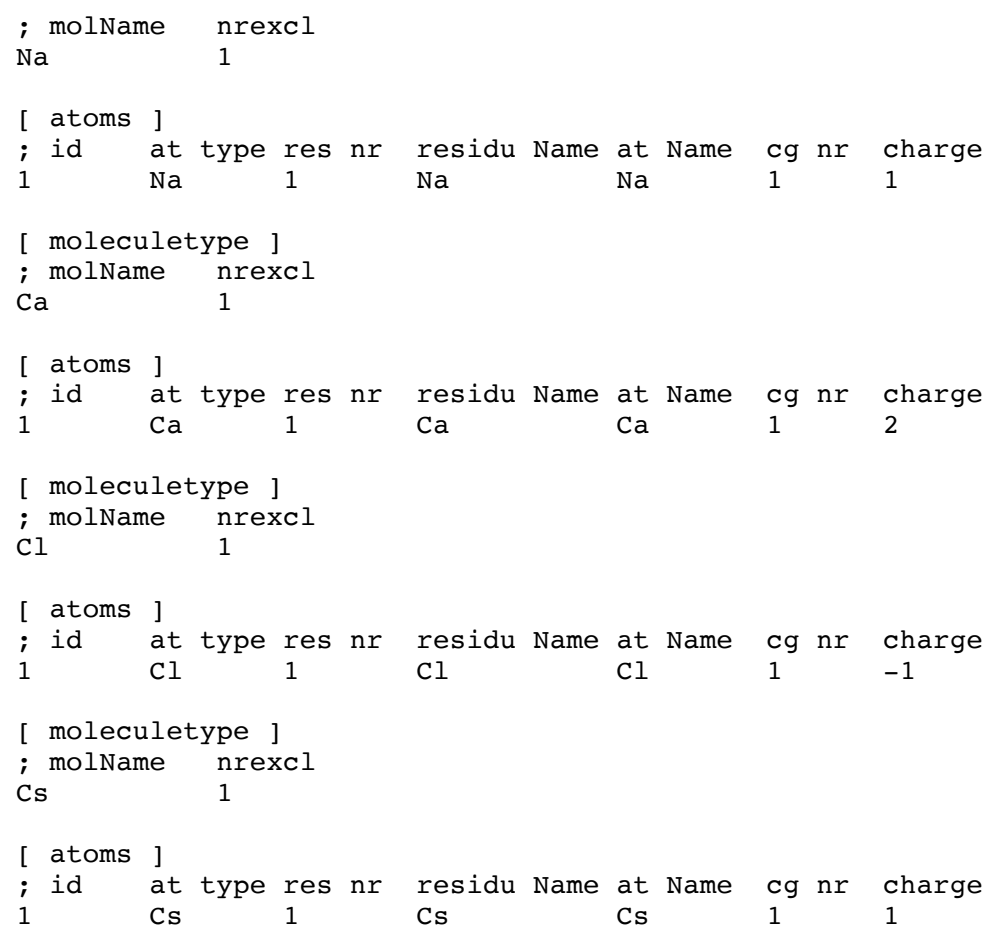

$\begin{array}{rrrrr}\text { ho } & 1 & \text { MON } & \text { HO1 } & 1 \\ \text { ho } & 1 & \text { MON } & \text { HO2 } & 2 \\ \text { ho } & 1 & \text { MON } & \text { HO3 } & 3 \\ \text { ho } & 1 & \text { MON } & \text { HO4 } & 4 \\ \text { ohs } & 1 & \text { MON } & \text { OHS1 } & 5 \\ \text { ohs } & 1 & \text { MON } & \text { OHS2 } & 6 \\ \text { oh } & 1 & \text { MON } & \text { OH1 } & 7 \\ \text { oh } & 1 & \text { MON } & \text { OH2 } & 8 \\ \text { obos } & 1 & \text { MON } & \text { OBS1 } & 9 \\ \text { obos } & 1 & \text { MON } & \text { OBS2 } & 10 \\ \text { ob } & 1 & \text { MON } & \text { OB1 } & 11 \\ \text { obos } & 1 & \text { MON } & \text { OBS3 } & 12 \\ \text { obos } & 1 & \text { MON } & \text { OBS4 } & 13 \\ \text { ob } & 1 & \text { MON } & \text { OB2 } & 14 \\ \text { ob } & 1 & \text { MON } & \text { OB3 } & 15 \\ \text { ob } & 1 & \text { MON } & \text { OB4 } & 16 \\ \text { ob } & 1 & \text { MON } & \text { OB5 } & 17 \\ \text { ob } & 1 & \text { MON } & \text { OB6 } & 18 \\ \text { ob } & 1 & \text { MON } & \text { OB7 } & 19 \\ \text { ob } & 1 & \text { MON } & \text { OB8 } & 20 \\ \text { ob } & 1 & \text { MON } & \text { OB9 } & 21 \\ \text { ob } & 1 & \text { MON } & \text { OB10 } & 22 \\ \text { ob } & 1 & \text { MON } & \text { OB11 } & 23 \\ \text { ob } & 1 & \text { MON } & \text { OB12 } & 24 \\ \text { ob } & 1 & \text { MON } & \text { OB13 } & 25 \\ \text { ob } & 1 & \text { MON } & \text { OB14 } & 26 \\ \text { ob } & 1 & \text { MON } & \text { OB15 } & 27 \\ \text { ob } & 1 & \text { MON } & \text { OB16 } & 28 \\ \text { mgo } & 1 & \text { MON } & \text { MGO1 } & 29 \\ \text { ao } & 1 & \text { MON } & \text { AO1 } & 30 \\ \text { ao } & 1 & \text { MON } & \text { AO2 } & 31 \\ \text { ao } & 1 & \text { MON } & \text { AO3 } & 32\end{array}$




\begin{tabular}{|c|c|c|c|c|c|c|c|c|c|c|}
\hline 33 & st & 1 & MON & ST1 & 33 & 2.1 & 28.09 & ; & qtot & -15.7 \\
\hline 34 & st & 1 & MON & ST2 & 34 & 2.1 & 28.09 & ; & qtot & $-13 \cdot 6$ \\
\hline 35 & st & 1 & MON & ST3 & 35 & 2.1 & 28.09 & ; & qtot & -11.5 \\
\hline 36 & st & 1 & MON & ST4 & 36 & 2.1 & 28.09 & ; & qtot & -9.4 \\
\hline 37 & st & 1 & MON & ST5 & 37 & 2.1 & 28.09 & ; & qtot & $-7 \cdot 3$ \\
\hline 38 & st & 1 & MON & ST6 & 38 & 2.1 & 28.09 & ; & qtot & -5.2 \\
\hline 39 & st & 1 & MON & ST7 & 39 & 2.1 & 28.09 & ; & qtot & -3.1 \\
\hline 40 & st & 1 & MON & $\mathrm{ST} 8$ & 40 & 2.1 & 28.09 & ; & qtot & -1 \\
\hline
\end{tabular}

\begin{tabular}{lllrr} 
[ bonds ] & \multicolumn{3}{l}{ funct } & \multicolumn{2}{l}{ length $\begin{array}{c}\text { force.c. } \\
i\end{array}$} & $j$ & & 0.1 & 463532.808 \\
1 & 5 & 1 & 0.1 & 463532.808 \\
2 & 6 & 1 & 0.1 & 463532.808 \\
3 & 7 & 1 & 0.1 & 463532.808
\end{tabular}

\section{REFERENCES}

(1) Cygan, R. T.; Liang, J.-J.; Kalinichev, A. G. Molecular Models of Hydroxide, Oxyhydroxide, and Clay Phases and the Development of a General Force Field. J. Phys. Chem. B 2004, 108, 1255-1266.

(2) Berendsen, H. J. C.; Postma, J. P. M.; van Gunsteren, W. F.; Hermans, J. Interaction Models for Water in Relation to Protein Hydration. In Intermolecular Forces, Pullman, B., Ed. D. Reidel: 1981; pp 331- 342.

(3) Berendsen, H. J. C.; Grigera, J. R.; Straatsma, T. P. The Missing Term in Effective Pair Potentials. J. Phys. Chem. 1987, 91, 6269-6271.

(4) Teleman, O.; Jönsson, B.; Engström, S. A Molecular Dynamics Simulation of a Water Model with Intramolecular Degrees of Freedom. Mol. Phys. 1987, 60, 193-203.

(5) Greathouse, J. A.; Durkin, J. S.; Larentzos, J. P.; Cygan, R. T. Implementation of a Morse Potential to Model Hydroxyl Behavior in Phyllosilicates. J. Chem. Phys. 2009, 130, 134713.

(6) Zeitler, T. R.; Greathouse, J. A.; Gale, J. D.; Cygan, R. T. Vibrational Analysis of Brucite Surfaces and the Development of an Improved Force Field for Molecular Simulation of Interfaces. J. Phys. Chem. C 2014, 118, 79467953.

(7) Pouvreau, M.; Greathouse, J. A.; Cygan, R. T.; Kalinichev, A. G. Structure of Hydrated Gibbsite and Brucite Edge Surfaces: DFT Results and Further Development of the Clayff Classical Force Field with Metal-O-H Angle Bending Terms. J. Phys. Chem. C 2017, 121, 14757-14771.

(8) Pouvreau, M.; Greathouse, J. A.; Cygan, R. T.; Kalinichev, A. G. Structure of Hydrated Kaolinite Edge Structures: DFT Results and Further Development of the Clayff Classical Force Field with Metal-O-H Angle Bending Terms. J. Phys. Chem. C 2019, 123, 11628-11638.

(9) Knapp, B.; Lederer, N.; Omasits, U.; Schreiner, W. vmdICE: A Plug-in for Rapid Evaluation of Molecular Dynamics Simulations Using VMD. J. Comput. Chem. 2010, 31, 2868-2873.

(10) Stukowski, A. Visualization and Analysis of Atomistic Simulation Data with OVITO-The Open Visualization Tool. Model. Simul. Mater. Sci. Eng. 2010, 18, 015012.

(11) Jamali, S. H.; Wolff, L.; Becker, T. M.; de Groen, M.; Ramdin, M.; Hartkamp, R.; Bardow, A.; Vlugt, T. J. H.; Moultos, O. A. OCTP: A Tool for On-the-Fly Calculation of Transport Properties of Fluids with the Order- $n$ Algorithm in LAMMPS. J. Chem. Inf. Model. 2019, 59, 1290-1294

(12) Holmboe, M. Atom: A Matlab Package for Manipulation of Molecular Systems. Clays Clay Miner. 2019, 67, 419-426.

(13) Åqvist, J. Ion-Water Interaction Potentials Derived from Free Energy Perturbation Simulations. J. Phys. Chem. 1990, 94, 8021-8024.

(14) Koneshan, S.; Rasaiah, J. C.; Lynden-Bell, R. M.; Lee, S. H. Solvent Structure, Dynamics, and Ion Mobility in Aqueous Solutions at $25^{\circ}$ C. J. Phys. Chem. B 1998, 102, 4193-4204.

(15) Li, P.; Roberts, B. P.; Chakravorty, D. K.; Merz, K. M. Rational Design of Particle Mesh Ewald Compatible Lennard-Jones Parameters for +2 Metal Cations in Explicit Solvent. J. Chem. Theory Comput. 2013, 9, 2733-2748.

(16) Li, P.; Song, L. F.; Merz, K. M. Systematic Parameterization of Monovalent Ions Employing the Nonbonded Model. J. Chem. Theory Comput. 2015, 11, 1645-1657. 